\title{
Recommandations sur la conception, le calcul, l'exécution et le contrôle des colonnes ballastées sous bâtiments et ouvrages sensibles au tassement
}

\author{
[O Le présent document vise à modifier et à compléter le \\ chapitre 8 de la norme NFP 11-212 (référence DTU 13,2 \\ ( Fondations profondes pour le bâtiment $x)$. \\ Il s'articule autour des chapitres suivants: le domaine \\ d'application, les dispositions constructives, les modes \\ opératoires, les principes de dimensionnement, \\ et les éléments de contrôle qu'il convient d'associer \\ au procédé de renforcement de sols par colonnes ballastées. \\ Ont participé à la rédaction du présent document : \\ P. Berthelot (Bureau Veritas) \\ C. Besson (Intrafor, président du SOFFONS) \\ M. Boucherie (Socotec) \\ L. Carpinteiro (Socotec) \\ N. Deryckere (Keller Fondations spéciales) \\ A. Frossard (Solétanche Bachy Pieux) \\ M. Glandy (Solétanche Bachy Pieux) \\ B. Pezot (Menard Soltraitement) \\ C. Poilpré (GTS) \\ J.P. Volcke (Franki Fondation) \\ Ont également participé aux discussions : Mme Altmayer \\ (Bureau Veritas), MM. Avoine et Blondeau (Apave), \\ M. Burban (Soletanche Bachy Pieux), M. Stoehr, \\ MM. Georget et Dhouib (Keller Fondations spéciales), \\ M. Pignerol (Intrafor), M. Touquet (SPIE Fondations). \\ Ce document a reçu l'aval du Comité français de \\ mécanique des sols (CFMS).
}

\section{Avant-propos \\ Jean Launay \\ Président du CFMS}

Les projets de renforcements de sols se sont multipliés les dernières années.

En particulier, les chantiers de colonnes ballastées ont connu un développement considérable.

Les professionnels (depuis le géotechnicien jusqu'au contrôleur technique) ont cependant été confrontés, d'une part, à une quasi absence de texte de référence, puisque le seul document sur le sujet est un chapitre du DTU 13.2 dont la rédaction remonte aux années 70 et, d'autre part, à une abondance de documents de conception dont les conclusions pouvaient être, sinon contradictoires, du moins non cohérentes en apparence.

Le CFMS a donc été saisi d'un projet de recommandations dont l'objectif déclaré est de constituer, entre autres, une mise à jour des dispositions constructives et un référentiel pour le dimensionnement des colonnes ballastées sous les ouvrages et constructions essentiellement de bâtiment sensibles aux tassements.

Le texte ci-après est le résultat des délibérations et constitue le socle sur lequel les professionnels des sols, particulièrement réunis dans le CFMS, mais aussi d'autres (bureaux d'études de structures, maîtres d'œuvre...) pourront s'appuyer pour confirmer l'usage des colonnes ballastées. 


\section{Préambule}

Les colonnes concernées par les présentes recommandations professionnelles sont constituées de matériaux granulaires, sans cohésion, mis en place par refoulement dans le sol et compactés par passes successives.

Commentaire. Ces colonnes ne comportent en particulier aucun liant sur leur hauteur. Dans la suite du texte, lexpressior a colonne ballastèe n s'entend pour la seule définition ci-dessus.

Les colonnes peuvent être réalisées en maillages réguliers ou variables, en lignes ou en groupes ou même de manière isolée.

Leur dimensionnement tient compte du type d'ouvrage à réaliser, de la nature des charges, des tolérances ou prescriptions relatives aux tassements absolus et différentiels, ainsi que de la nature du sol à traiter.

Le but de toute réalisation de colonnes ballastées est de conférer au sol de nouvelles caractéristiques, générales et/ou locales sous l'ouvrage à construire, afin que les différents éléments d'infrastructure de celui-ci (semelles isolées ou filantes, radiers, dallages, ouvrages en terre...) aient un comportement prévisible, justifiable et compatible avec les règlements et tolérances s'appliquant à la structure de l'ouvrage et à son exploitation.

Le traitement d'un sol par colonnes ballastées conjuque les actions suivantes, dont une seule ou plusieurs peuvent être recherchées:

- amélioration de la portance,

- réduction des tassements,

- homogénéisation des caractéristiques géotechniques, - augmentation de la vitesse de consolidation par la création d'éléments drainants,

- augmentation des caractéristiques équivalentes du massif de sol traité (la résistance au cisaillement horizontal, langle de frottement interne et les paramètres de déformation).

Une colonne ballastée est un procédé d'amélioration de sol: ce n'est ni un élément de fondation, ni une fondation profonde.

La fondation d'un ouvrage reposant sur un sol traité par colonnes ballastées est toujours de type superficiel semelle filante ou isolée, radier, dallage. Il peut aussi s'agir de la «fondation» d'un ouvrage en terre.

C'est la maitrise du comportement de la fondation superficielle qui est recherchée.

La faisabilité du traitement de sol doit faire l'objet d'une analyse par le géotechnicien de l'opération qui, par ailleurs, fournira ou avalisera les hypothèses de calcul; selon sa mission, il peut être amené à effectuer un prédimensionnement de l'opération.

Commentaire. Il est rappelé que l'étude géotechnique pour des colonnes ballastées doit comprendre la reconnaissance des terrains à traiter maís aussi de ceux susceptibles de tasser sous l'épaisseur traitée.

Il est souhaitable que le maitre d'ouvrage associe le géotechnicien au projet dans le cadre des missions successives définies par la norme NFP. $94-500$.

\section{Domaine d'application}

\section{1}

\section{Par types d'ouvrages}

Les utilisations les plus fréquentes des traitements par colonnes ballastées concernent des ouvrages oû existent des dallages et radiers recevant des charges surfaciques et susceptibles d'accepter des tassements: - halls de stockage,

- bâtiments industriels et commerciaux,

- silos et réservoirs de toute nature.

- ouvrages hydrauliques étanches (réservoirs, station d'épuration).

Par extension, on peut les utiliser sous d'autres types d'ouvrages dans la mesure où les déformations résiduelles du sol traité et du sol sous-jacent sont compatibles avec la structure de l'ouvrage sous l'exploitation et les prescriptions techniques associées:

- en génie civil (routes, remblais, ouvrages d'art, murs de soutènement) ou maritime (renforcement de fonds marins, lacustres ou fluviaux);

-sous fondations superficielles de bâtiments.

Elles peuvent également être utilisées dans des remblais hétérogènes non évolutifs, où un traitement systématique avec un maillage régulier et adapté permet d'en améliorer et/ou homogénéiser les caractéristiques, afin de les rendre aptes à fonder superficiellement les ouvrages projetés.

\section{2}

\section{Utilisation en zone sismique}

Il est également possible d'utiliser les colonnes en zone sismique où elles peuvent contribuer à la diminution du potentiel de liquéfaction des sols.

L'annexe I reprend les dispositions à appliquer dans ce cas.

\section{3}

\section{Limites d'utilisation sous structures sensibles aux déformations}

Les colonnes ballastées ne doivent pas être utilisées dans des terrains présentant des risques de perte dans le temps des caractéristiques volumétriques et/ou mécaniques, notamment les décharges d'ordures ménagères, les tourbes et, de manière générale, les sols présentant une perte au feu supérieure à $5 \%$, au sens de la norme XP 94-047.

Commentaire. Il existe aussi la norme NF P 94-055 qui définit une méthode chimique pour dêterminer la teneur pondérale en matière organique d'un matériau.

Les sols fortement compressibles (vases et argiles molles) d'épaisseur supérieure à $0,50 \mathrm{~m}$ et présentant des caractéristiques faibles $\left(\mathrm{c}_{\mathrm{u}}<20 \mathrm{kPa}\right.$ ou $\left.\mathrm{q}_{c}<300 \mathrm{kPa}\right)$ nécessiteront une étude particulière et des dispositions constructives spécifiques: par exemple, préchargement, consolidation. 


\section{Mode opératoire}

\section{1}

\section{Méthodes et matériels}

Les deux procédés suivants satisfont à la définition donnée en préambule.

Les colonnes pilonnées: voir le lexique pour le descriptif détaillé.

Les colonnes vibrées:

- si l'on utilise le lançage à l'air, on parle de voie sèche: -si l'on utilise le lançage à l'eau, on parle de voie humide.

Commentaire. Tout autre procédé doit faire l'objet d'un cahier des charges.

Le choix de l'outil, de ses caractéristiques et de la méthode de réalisation dépend étroitement de la nature et de l'état de saturation du sol, du but recherché et des caractéristiques des matériaux d'apport. Il appartient à l'entreprise, en fonction de son savoir-faire et de son expérience, de choisir l'outil et le procédé le mieux adaptés dans chaque cas.

\section{2}

\section{Préforages}

La traversée de couches compactes ou d'obstacles peut être facilitée par un forage préalable, avec ou sans extraction de terrain.

Tout volume excavé doit être rempli et compacté par le matériau de la colonne.

\section{3}

\section{Matériaux d'apport}

Les matériaux d'apport doivent être de qualité et de granulométrie contrôlées et les plus homogènes possibles.

Le choix se portera sur des graves naturelles, roulées ou concassées.

Commentaire. Sauf dossier spécifique démontrant l'absence de désordres à court et à long termes (gonflement, pollution, réaction physico-chimique,...), les matériaux recyclés ne sont pas adimis.

Les caractéristiques minimales des matériaux d'apport sont les suivantes:

$-\mathrm{LA}<35$

$-\mathrm{MDE}<30$

$-\mathrm{LA}+\mathrm{MDE}<60$

Commentaire. LA: essai Los Angeles, norme NF P 18573 , MDE: essai Micro Deval, norme NF P 18572.

La granulométrie dépend essentiellement du matériel. Les vibreurs avec alimentation en pied y sont plus sensibles: une granulométrie inadaptée est source de bouchons dans le tube.

On peut retenir les valeurs indicatives suivantes: - vibreur à tube latéral de remplissage par le bas : en terme de fuseau granulométrique, le plus couramment utilisé est le fuseau $8 / 4$

- autres procédés: en terme de fuseau granulométrique, celui qui est le plus couramment utilisé est le fuseau 20/75.

Le critère de propreté au sens de la norme NFP 18591 est le suivant: le passant inférieur à $80 \mu \mathrm{m}$ est inférieur à $5 \%$.

4

\section{Dispositions constructives}

\section{1}

\section{Diamètre des colonnes ballastées}

Le diamètre des colonnes ballastées dépend: - de l'outil utilisé et de l'adéquation de ce choix au terrain rencontré

- des terrains traversés et de leurs caractéristiques; - de l'énergie totale dépensée (puissance mise en œuvre, poussée verticale éventuelle et temps passé).

Le diamètre de la colonne peut varier sur sa hauteur, en fonction des différences de résistance des couches traitées.

Commentaire. Les diamètres usuels par voie sèche sont compris entre 50 et $80 \mathrm{~cm}$.

Toutes choses égales par ailleurs, le diamètre de la colonne est plus important par voie humide que par voie sèche, du fait de l'extraction de sol produite par le lançage à l'eau.

\section{2}

\section{Interface sol traité/structure}

Si l'élément de fondation a une inertie insuffisante pour répartir sa charge de façon homogène sur la maille élémentaire, il est nécessaire de disposer un matelas de répartition entre l'élément de fondation et le sol traité : ce matelas a pour rôle d'assurer la répartition complémentaire des charges.

Si l'on utilise l'action drainante des colonnes, il faut disposer d'une couche drainante (avec exutoire) au toit des colonnes.

Commentaire. En pratique, hors zone sismique, les semelles isolées et filantes ainsi que les raciers ne nécessitent en généra aucun matelas, contrairement aux dallages, armés ou non

Par ailleurs, le fait de traiter un sol par incorporation de colonnes ballastées n'améliore pas, sauf exceptions, le comportement du terrain en place en surface entre colonnes où il n'y a donc pas augmentation a priori des valeurs du module EV2 ou celles du coefficient de Westergaard.

\section{3}

\section{Exécution des semelles et radiers}

Ceux-ci doivent être réalisés de la même manière que dans un sol naturel, ils peuvent être coulés à pleine fouille ou coffrés.

Lorsque la base de la semelle est à moins de $50 \mathrm{~cm}$ sous le niveau de la plate-forme de travail, la tête de la 
colonne doit être recompactée pour garantir les caractéristiques prises en compte dans les calculs.

Commentaire. Ce recompactage peut faire partie du recompactage de la plate-forme.

\section{4}

\section{Exécution du matelas de répartition}

L'épaisseur minimale d'un matelas de répartition en matériaux granulaires est supérieure à 40 cm.

Dans le cas des dallages, la partie supérieure du matelas de répartition a au moins les caractéristiques d'une couche de forme, au sens du document GTR92.

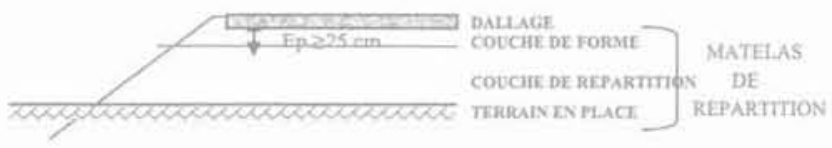

Commentaire. Dans le cadre de ce document, on retiendra en particulier pour la couche de forme que:

- les matériaux de classe F (norme NF P 11-300) ne sont pas admis:

- l'épaisseur minimale est de $25 \mathrm{~cm}$;

- le module d'élasticité est supérieur à $50 \mathrm{MPa}$ :

Il appartient au concepteur de l'amélioration de sol de définir l'épaisseur et les caractéristiques minimales de ce matelas par rapport au sol traité.

Commentaire. Il est rappelé à ce propos que les critères intervenant pour le calcul d'un matelas de répartition sont, d'une part, sa résistance au poinçonnement (si nécessaire), d'autre part, l'épaisseur et le module d'élasticité.

Le concepteur du dallage devra vérifier que l'épaisseur et les caractéristiques du matelas sont suffisantes vis-à-vis des impératifs résultant des sollicitations du dallage, notamment le poinçonnement, et qu'elles restent supérieures aux minima requis par les normes d'exécution de ces dallages.

La couche de répartition peut être mise en place totalement ou partiellement avant exécution des colonnes ballastées: elle sert alors de plate-forme de travail.

La couche de forme peut également être réalisée avant les colonnes ballastées.

Cependant, le reprofilage, le compactage final, le retraitement et éventuellement le complément en épaisseur devront être réalisés après les colonnes ballastées, afin d'assurer un nivellement et des caractéristiques conformes aux éléments du projet.

\section{5}

\section{Disposition des colonnes ballastées}

\section{9.}

\section{Ouvrages à charges réparties uniformes}

Dans le cas d'ouvrages à charges réparties uniformes, les colonnes sont disposées selon un maillage régulier, gênéralement carré ou triangulaire.

Le maillage dépend des caractéristiques géotechniques et des charges appliquées.
Commentaire 1. Dans certaines circonstances de caractéristiques géotechniques et/ou de chargement, une partie des ouvrages peut ne pas reposer sur un terrain traité dans la mesure où les tassements absolus et différentiels entre zones traitées ou chargées différemment restent compatibles avec la structure et son exploitation.

Commentaire 2. Sauf justifications particulières et hors zone sismique, il n'est pas nécessaire de disposer des colonnes á l'extérieur de l'emprise de l'ouvrage concerné.

\section{x.s.5. \\ Semelles filantes et isolées}

Le dimensionnement des semelles est fonction à la fois de la contrainte admissible et du tassement admissible après traitement de sol.

Le maillage résulte du critère le plus défavorable.

Commentaire. Au sein d'un méme ouvrage, it est parfaitement envisageable qu'il y ait des semelles courantes sous lesquelles le sol a été amélioré et des semelles faiblement chargées reposant sur le sol naturel. C'est le respect de l'homogénéité des tassements des semelles soumises à des charges dissemblables qui peut autoriser cette situation (exemple: ossature secondaire support de bardage des halls industriels).

Pour des colonnes isolées ou des files uniques de colonnes, en l'absence de vérification adaptée, le débord de la semelle vis-à-vis de la position théorique du nu extérieur des colonnes doit être au moins égal à la tolérance d'exécution définie au $\$ 4.9$.

Commentaire. La vérification consiste à s'assurer que, même si la colonne n'est pas intégralement sous le massif (éventuellement avant exécution et obligatoirement après le récolement si celui-ci démontre un excentrement), les résultats en terme de déformation et de contrainte maximale dans la colonne et dans le sol restent admissibles.

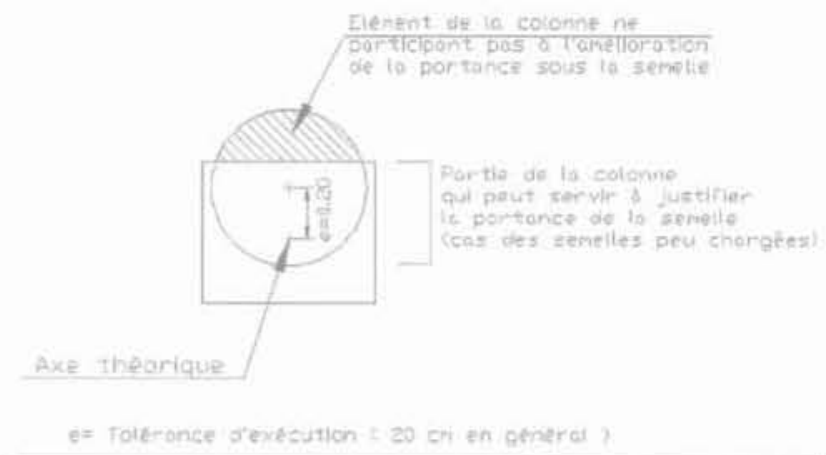

CAS DE COLDNNES DEBDRDANT DES SEMELLES

Pour des colonnes non alignẻes, il est recommandé d'adopter la même disposition (cébord de la semelle au moins égal à la tolérance d'exécution). Dans le cas contraire, il faut justifier le tassement et la contrainte dans les colonnes si les colonnes ne respectent pas l'implantation théorique.

Commentaire 1. On trouvera en annexe II quelques dispositions types.

Commentaire 2. Sous les semelles filantes, la disposition des colonnes en quinconce limite l'incidence des excentrements.

\section{6}

\section{Mailles de référence maximales}

Pour qu'un sol puisse être considéré comme traité par des colonnes ballastées, et quelle que soit l'action 
recherchée, la maille de référence la plus grande doit ètre de $9 \mathrm{~m}^{2}$, d'une part, et, le taux de substitution doit être supérieur à $3 \%$, d'autre part.

Pour une semelle filante comportant une seule rangée de colonnes et dépourvue de matelas de répartition, l'entraxe maximal sans justification spécifique est de $2,5 \mathrm{~m}$.

\section{Mailles de référence minimales}

La maille de référence minimale est de $2,4 \mathrm{~m}^{2}$.

Pour les semelles filantes et les groupes de 2 à 5 colonnes, l'espacement entre axes de colonnes n'est pas inférieur à $1,5 \varnothing$ et $1,20 \mathrm{~m}$.

Commentaire 1. Un taux de substitution élevé induit un risque de soulèvement des plates-formes.

Commentaire 2. Des mailles plus étroites, sans ètre inférieures à $1,5 \mathrm{~m}^{2}$, sont possibles mais nécessitent une étude spécifique de faisabilité.

Commentaire 3 . Pour des colonnes réalisées par lançage á l'eau, procédé peu utilisé en site terrestre du fait dé l'extraction de terrain généré par le procédé, il est possible de réaliser des colonnes ballastées de diamètre important $(1,00$ à $1,20 \mathrm{~m})$ plus rapprochées que par voie sèche.

\section{8}

\section{Colonnes ballastées sous un remblai support de fondations}

On peut traiter par incorporation de colonnes ballastées tous les types de sols entrant dans le domaine d'application, et ensuite mettre en ceuvre sur le so! amélioré des remblais de rehaussement ou de substitution, réalisés avec des matériaux nobles de qualité contrôlée, mis en œuvre de manière soignée avec un contrôle rigoureux de leur compactage, par exemple selon les recommandations LCPC-COPREC de 1980

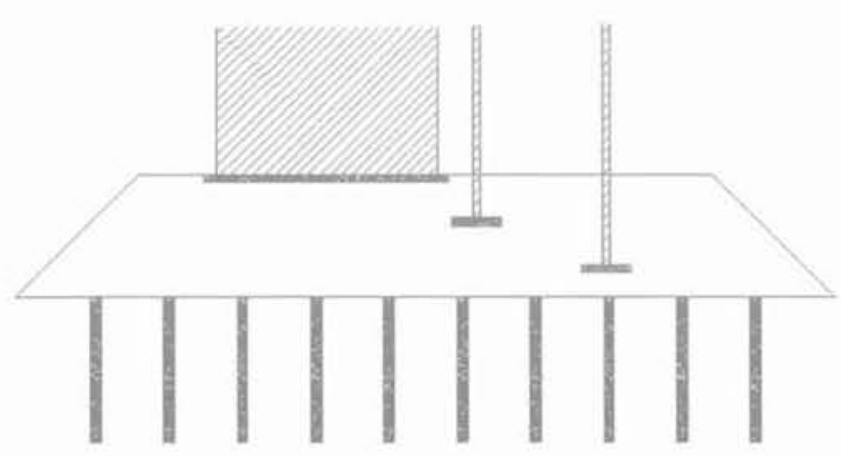

La disposition des colonnes tient compte de la superposition des contraintes générées d'une part par le remblai lui-même et, d'autre part, par les différents éléments de structure qui sont fondés dans son épaisseur; il faut justifier en particulier cette disposition si la hauteur du remblai est inférieure au demi-côté de la maille de référence.

\section{9}

\section{Tolérances d'exécution}

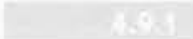 \\ Maillage en réseau
}

La colonne ballastée est une inclusion donnant, grâce à ses caractéristiques propres, de nouvelles caractéristiques équivalentes à la maille élémentaire de sol traité, dont elle occupe le centre.

Dans ce cas, il n'existe pas de notion d'icexcentrement telle que celle-ci apparait pour des éléments ponctuels de fondation.

\section{(4098) \\ Colonnes sous semelles}

Les semelles isolées et filantes reposant sur le sol amélioré doivent être justifiées comme des éléments de fondation.

La tolérance d'exécution des colonnes ballastées est alors de $\pm 20 \mathrm{~cm}$.

Commentaire. Cette valeur peut être difficile à apprécier du fait de la forme irrégulière des colonnes lorsqu'elles sont vibrées.

Au-delà de cette valeur, l'entreprise doit justifier que le comportement de la semelle reste compatible avec la structure de l'ouvrage; s'il y a une couche de répartition sous la semelle, on tient compte de ses caractéristiques pour cette justification.

Commentaire. Les ouvrages surmontant les colonnes sont concus de telle sorte qu'ils ne soient pas à modifier tant que les écarts d'implantation sont inférieurs aux tolérances d'exé. cution.

Le choix de tolérances plus faibles est laissé à l'initiative de l'entreprise mais celle-ci doit alors s'assurer de leur strict respect; cette tolérance peut ne pas ètre la mème dans les deux directions.

\subsection{0}

\section{Coefficient réducteur sur les volumes de matériaux incorporés}

Il existe une différence entre le volume théorique des colonnes réalisées et le volume de matériau approvisionné et donc foisonné sur le site. Cette différence provient, d'une part, des pertes à l'exécution (dont les hors-profils variables en fonction de l'étreinte latérale) et, d'autre part, du compactage du matériau dans la colonne.

Généralement, on admet les rapports suivants du volume approvisionné au volume théorique:

- colonnes vibrées avec alimentation en tête, sous l'eau; 1,3 à 1,5 ;

-colonnes avec incorporation en pied, à l'air: 1,2. 


\section{Dimensionnement}

\section{1}

\section{Préalable : éléments d'information nécessaires au calcul}

Le comportement d'un renforcement de sol par colonnes ballastées ne peut s'analyser sans une bonne connaissance:

- de la nature et des caractéristiques du sol traité et sous-jacent: coupes de sondages, résultats des essais mécaniques en laboratoire ou en place... :

- de la nature, des caractéristiques et des méthodes d'exécution de la fondation proprement dite;

- des sollicitations de service ou exceptionnelles:

- de la nature et du comportement de la structure à fonder.

Les règles de calcul du présent document ne prennent pas en compte d'amélioration du sol entre les colonnes.

Commentaire. L'exécution des colonnes peut améliorer les caractèristiques de certains sols : il est possible de prendre en compte des caractéristiques « améliorées $x$ à condition de vérifier par des essais appropriés qu'elles sont atteintes.

\section{2}

\section{Critères de dimensionnement}

Le dimensionnement des colonnes ballastées est indissociable des caractéristiques du sol à traiter et du procédé de mise en œuvre.

Les justifications à apporter dans la note de calculs se rapporteront dans le cas général aux deux critères suivants:

- charge admissible globale sur le sol amélioré après traitement et justification des différents types de fondations vis-à-vis de la rupture:

- tassement absolu des divers éléments de structure au sein d'un même ouvrage, justification des tassements différentiels au sein de la structure ou entre structure et dallage, en fonction des tolérances admissibles propres à chaque ouvrage et des règlements en vigueur.

Lorsque d'autres actions sont recherchées, la justification de ces effets doit être alors produite:

- dans le cas de la liquéfaction des sols, il convient de démontrer que les colonnes ont réduit ce risque;

- si l'effet drainant est recherché pour accélérer la consolidation, un calcul du temps de consolidation est établi;

- dans le cas de la stabilisation de talus, le calcul porte sur la sécurité obtenue vis-à-vis des glissements circulaires.

\section{3}

\section{Hypothèses}

Les contraintes d'exploitation de l'ouvrage (charges maximales et déformations admissibles) doivent être définies dans les documents particuliers du marché.
Les hypothèses concernant le sol sont les caractéristiques géotechniques mentionnées dans l'étude de sol: stratigraphie, nature des sols, hydrologie, caractéristiques rhéologiques et mécaniques (module d'Young, coefficient de Poisson, étreinte latérale, coefficient de compressibilité $\mathrm{Cc}$, indice des vides...) de toutes les couches de sol.

Commentaire. Au cas où les donnèes résulteraient de corrélations, il conviendra d'avoir l'accord écrit du géotechnicien sur la validité des hypothèses considérées.

Les hypothèses concernant les colonnes ballastées, notamment quant à leur module de déformation, diamètre, longueur, dépendent étroitement du matériel de mise en œuvre choisi et des performances de celui-ci.

Les valeurs usuelles des paramètres autres que géométriques, et entrant dans les calculs, sont les suivantes; pour les matériaux décrits au $\$ 3.3$ et correctement mis en œuvre:

-module d'Young

(moyenne sur le volume de la colonne) $E_{\text {col }}=60 \mathrm{MPa}$ -angle interne intergranulaire

(matériau roulé)

(matériau concassé)

- coefficient de Poisson

- poids volumique du matériau

en place, saturé

$\varphi_{\mathrm{c}}^{\prime}=38$ degrés $\varphi_{c}^{\prime \prime}=40$ degrés $v_{\text {col }}=1 / 3$

$\gamma_{\mathrm{cop}}=21 \mathrm{kN} / \mathrm{m}^{3}$

Commentaire. Des valeurs différentes, supérieures ou inférieures, sont possibles mais devront être justifiées par des essais appropriés.

\section{4}

\section{Contraintes maximales admissibles dans les colonnes}

Le calcul de la contrainte maximale admissible consiste d'abord à déterminer la contrainte verticale de rupture $\mathrm{q}_{r}$ d'une colonne isolée à partir des caractéristiques des colonnes et du sol après traitement et ce selon les schémas de rupture possibles suivants (Fig. a, bet c):

- rupture par expansion latérale (critère souvent dimensionnant);

- rupture par cisaillement gênéralisé (rupture rare, cas des colonnes courtes);

- rupture par poinçonnement (colonnes flottantes).

\subsection{4}

\section{Rupture par expansion latérale}

Par analogie aux conditions triaxiales, la contrainte de rupture effective $\mathrm{q}_{\mathrm{re}}$ par expansion latérale est donnée en fonction de l'étreinte maximale latérale $\sigma_{\text {tmax }}^{\prime}$ par:

$$
\mathrm{q}_{t e}=\tan ^{2}\left(\pi / 4+\varphi_{c}^{\prime} / 2\right) \cdot \sigma_{\text {hmax }}^{\prime}
$$

Greenwood (1970)

La valeur de l'étreinte latérale résulte du rapport géotechnique; elle est déterminée à partir d'essais de laboratoire (essais triaxaux) ou d'essais in situ (pressiomètre, pénétromètre statique, scissomètre...).

Commentaire. Par exemple, dans le cas du pressiomètre, on retient:

$$
\sigma_{\operatorname{timax}}^{*}=p_{1}^{*}
$$


Si on a en outre $\varphi^{\prime}$, de l'ordre de 38 degrés, il vient alors:

$$
q_{\mathrm{rs}}=4 \mathrm{p}_{1}^{*}
$$

où $\mathrm{p}_{1}^{\prime}$ est la pression limite nette équivalente.

\section{(5.45:}

\section{Rupture par cisaillement généralisé}

La rupture par cisaillement généralisé peut être étudiée lorsque les caractéristiques de la colonne sont relativement proches de celles du sol. Ce cas est peu fréquent et le calcul correspondant n'est pas présenté ici (cf, Soyez, 1985).

\section{$5 \times 43$}

\section{Rupture par poinçonnement (colonnes flottantes)}

La contrainte verticale régnant au sein de la colonne est maximale en tête de la colonne et décroît en fonction de la profondeur (Soyez, 1985). Dans un milieu caractérisé par la cohésion non drainée $c_{u^{\prime}}$ la contrainte verticale de rupture vis-à-vis du poinçonnement est calculée selon la formule suivante:

où:

$$
\mathrm{q}_{\mathrm{TP}}=9 \cdot \mathrm{c}_{\mathrm{u}}+\mathrm{L}_{\mathrm{c}} \cdot\left(2 \mathrm{c}_{\mathrm{u}} / \mathrm{R}_{\mathrm{c}}-\gamma_{\mathrm{c}}\right)
$$

$\gamma_{c}$ : poids volumique de la colonne:

$\mathrm{L}_{c}$ : longueur de la colonne;

$\mathrm{R}_{\mathrm{c}}$ : rayon moyen de la colonne.

Commentaire. En pratique, on élimine le risque de poinçonnement en donnant à la colonne une longueur supérieure à la valeur minimale qui équilibre la résistance du sol:

-sous sollicitations ELU: $L_{c} \geq R_{c}\left[\left(\gamma_{E w}, \sigma_{0 H U} / c_{v}\right)-9\right] / 2$

-sous sollicitations ELS: $L_{c} \geq F_{c}\left[\left(\gamma_{\text {EIS. }} \cdot \sigma_{\text {eEs }} / c_{\mathrm{u}}\right)-9\right] / 2$

$\sigma_{0}$ est la contrainte en tête de colonne,

$\gamma_{\text {EUU }}$ (respectivement $\gamma_{E L S}$ ) vaut 1,5 (respectivement 2).

\section{Sach}

\section{Contraintes admissibles dans les colonnes à l'ELS}

La contrainte verticale admissible $\mathrm{q}$, dans la colonne est obtenue par application d'un coefficient de sécurité de 2 sur la contrainte verticale de rupture q.

La contrainte admissible retenue est la plus petite des valeurs: $0,8 \mathrm{MPa}$ (valeur plafonnée par la norme NF P 11 212, DTU 13.2) et la contrainte admissible calculée $q_{\text {a, }}$ soit:

$$
\mathrm{q}_{\mathrm{a}}=\min \left(0,8 \mathrm{MPa} ; \mathrm{q}_{\mathrm{r}} / 2\right)
$$

$\operatorname{avec} \mathrm{q}_{\mathrm{r}}=\min \left(\mathrm{q}_{\mathrm{re}} ; \mathrm{q}_{\mathrm{rp}}\right)$

\subsection{5}

\section{Contraintes dans les colonnes à l'ELU}

La contrainte maximale de calcul $\mathrm{q}_{\mathrm{a} \text { esu }}$ dans la colonne est obtenue par application d'un coefficient de sécurité de 1,5 sur la contrainte verticale de rupture $q_{f}$ :

$$
\mathrm{q}_{\text {aEt } \mathrm{U}}=\mathrm{q}_{\mathrm{r}} / 1,5
$$

Commentaire. Ceci revient à affecter un coefficient de 1,33 à la contrainte admissible à l'ELS :

$$
\mathrm{q}_{\text {anqu }}=1,33 \mathrm{q}_{\text {a }}
$$

\section{$5 \times 46$}

Contraintes dans les colonnes sous séisme

Voir en annexe I.

\section{5}

\section{Évaluation des contraintes dans les colonnes et des tassements}

Les méthodes ci-après ne sont a priori valides que si la surcharge apportée au sol entre les colonnes (calculée par lesdites méthodes) reste inférieure à la contrainte admissible pour le sol non traité.

Commentaire. Dans le cadre de la méthode pressiométrique, on doit vérifier:

$$
\mathrm{q}_{2}<\mathrm{k}^{*} \mathrm{p}_{\mathrm{i}} / 2+\mathrm{q}_{\alpha}^{\prime} \text { et } \mathrm{q}_{\mathrm{col}}<\mathrm{q}_{\mathrm{m}}
$$

$p, / 2$ étant la valeur usuelle de limite de validité des formules de tassement selon Ménard.

D'autres méthodes d'utilisation plus délicate sont également disponibles; il convient surtout de retenir les ordres de grandeur obtenus par les cas particuliers ci-après.

\section{B.5.}

\section{Etude des cas particuliers des dallages} et radiers soumis à un chargement uniforme infini

L'approche simplifiée est basée sur les hypothèses d'élasticité du sol et la conservation des sections planes horizontales; elle suppose également que les colonnes sont arrêtées sur une couche plus compacte.

Après réalisation des colonnes, le tassement de chaque couche i au centre de l'ouvrage s'écrit :

$$
w_{i}=\frac{h_{1} \times \sigma_{t}}{a_{i} E_{c o l}+\left((1-a) E_{s i}\left(1-v_{s i}\right) /\left(1-v_{s i}-2^{*} v_{s i}^{2}\right)\right)}
$$

c)

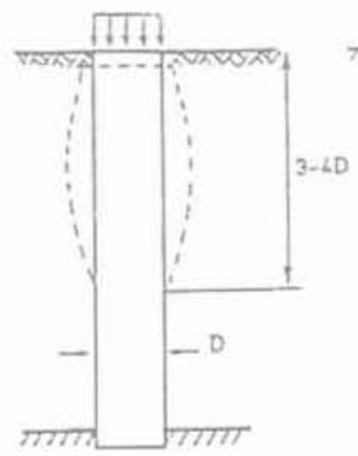

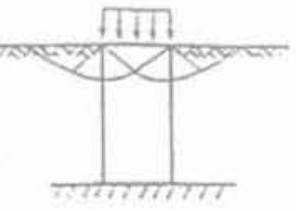

a) ruplure por expansion lotérole de lo colanne

b) ruplure por cisolilement générolisé

c) ruplute por poinçonnement icolonne flotionte)

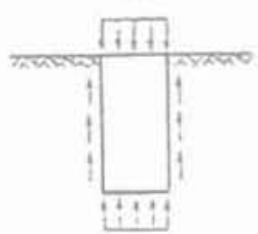

REVUE PANCANE DE GEOT 
et la valeur de la contrainte dans la colonne au niveau de la couche i $\left(\sigma_{\mathrm{c}}\right)$ peut être donnée par :

$$
\sigma_{c i}=\frac{E_{c i t} \times \sigma_{f}}{\left.a_{i} E_{c o t}+1\left(1-a_{j}\right) E_{s i}\left(1-v_{s i}\right) /\left(1-v_{s i}-2^{*} v_{s i}^{2}\right)\right)}
$$

où:

$a_{1}$ : pourcentage d'incorporation (rapport des sections), dans la couche i considérée;

$\mathrm{E}_{\text {col }}$ : module d'Young de la colonne:

$\mathrm{E}_{\mathrm{si}}$ : module d'Young de la couche i considérée;

$v_{s 1}$ : coefficient de Poisson de la couche i considérée;

$\sigma_{1}$ : contrainte verticale moyenne apportée par l'ouvrage:

$\mathrm{h}_{1}$; épaisseur de la couche i.

Commentaire, Dans le cas où on dispose d'essais pressio-

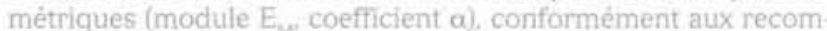
mandations dé la Société internationale de mécanique des sols e de géotechnique, on assimile le rapport $E_{3} / \alpha$ au module cedomé. trique

Dans l'hypothèse classique d'une valeur du coefficient de Poisson de 1/3, le module d'Young du sol Es est alors égal aux 2/3 du module cedométrique.

Les formules précédentes deviennent:

$$
w_{i}=\frac{h_{i} \times a_{\mathrm{r}}}{a_{i} E_{\mathrm{cos}}+\|\left(1-a_{)}\right) E_{k d} / a_{i} \mid}
$$

et la contrainte en tète de colonne:

$$
\sigma_{\mathrm{f}}=\frac{E_{\mathrm{aj}} \times a_{1}}{a_{i} E_{c o u}+\left|\left(1-a_{)}\right) E_{a d} / a_{1}\right|}
$$

Commentaire. Les règles pressiométriques peuvent être d'application délicates dans les argiles molles saturées.

Il convient alors de vérifier que cette contrainte reste inférieure aux maxima admissibles tels que définis dans l'alinéa 5.3.1:

$$
\sigma_{c}<\mathrm{q}_{\mu}
$$

et que le tassement total ( $\Sigma$ w; augmenté le cas échéant du tassement des couches situées sous la base des colonnes) reste inférieur aux valeurs fixées par les conditions d'exploitation.

Commentaire. Si les charges varient par zones et/ou si la stratigraphie n'est pas homogène, il faut vérifier aussi que les tassements differentiels sont acceptables

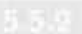

\section{Étude du cas particulier des colonnes sous semelle à charge verticale centrée}

L'approche simplifiée est basée sur les hypothèses d'élasticité du sol et la conservation des sections planes horizontales; elle suppose également que les colonnes sont arrêtées sur une couche plus compacte.

\subsubsection{Charges ELS}

Les étapes de calcul sont les suivantes, pour une semelle donnée (de surface $S_{5}=B{ }^{*} L$ ), reposant sur $n$ colonnes (de section unitaire $S_{c o l}$ ), réputées non flottantes, sous une surcharge (surfacique) $\mathrm{q}_{\mathrm{El.5}}$ (cf. tableau page suivante).

\subsubsection{2, Charges ELU}

On doit vérifier: $\left(n . S_{\text {col }} q_{a f L u}+\left(S_{s}-n \cdot S_{\text {col }}\right) q_{u}^{\prime} / 2\right)>$ $\mathrm{q}_{\mathrm{EI}, \mathrm{U}} \mathrm{S}_{\mathrm{s}}$

Commentaire, Si on a besoin des déformations à IELU, on peut utiliser en première approche la méthode décrite pour l'EL.S

\section{Sollicitations autres que les efforts verticaux centrés}

Soit une semelle de dimensions B $\times \mathrm{L}$, soumis à un torseur d'efforts dont les éléments au centre de la base sont la charge verticale $Q(Q=q \times B \times L)$, et les moments $\mathrm{M}_{\mathrm{x}}$ et $\mathrm{M}_{\mathrm{y}}$; L est parallèle à l'axe des $\mathrm{X}$.
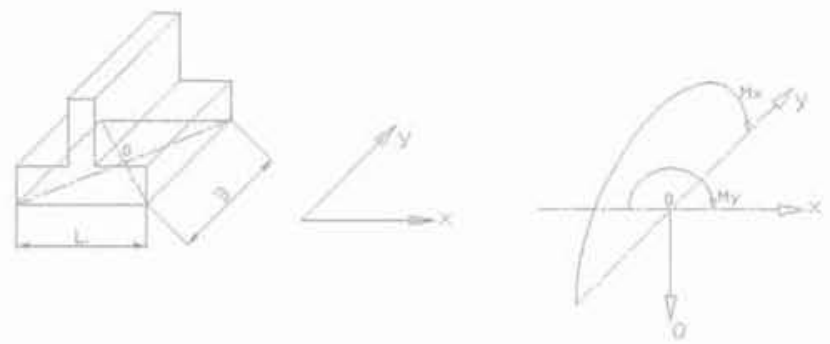

On impose qu'il y ait des colonnes sur plus d'un axe parallèle:

- à l'axe des $x$ dès que $M_{x} / Q>B / 6$;

- à l'axe des y dès que $M / Q>L / 6$.

Les méthodes décrites au $\$ 5.8$ abordent de différentes façons la question; nous exposons ci-après une méthode simplifiée.

\section{3. . \\ Méthode simplifiée}

On majore la valeur de $M_{x}$ d'un facteur $\psi_{x}$ qui vaut: $-1,25$ s'il y a des colonnes sur plusieurs axes parallèles à l'axe des $x$;

- et 1,5 dans le cas contraire (toutes les colonnes alignées sur l'axe des $\mathrm{x}$ ).

On majore la valeur de $M_{y}$ d'un facteur $\psi_{y}$ qui vaut $-1,25$ s'il y a des colonnes sur plusieurs axes parallèles à l'axe des y:

- et 1,5 dans le cas contraire (toutes les colonnes alignées sur l'axe des y).
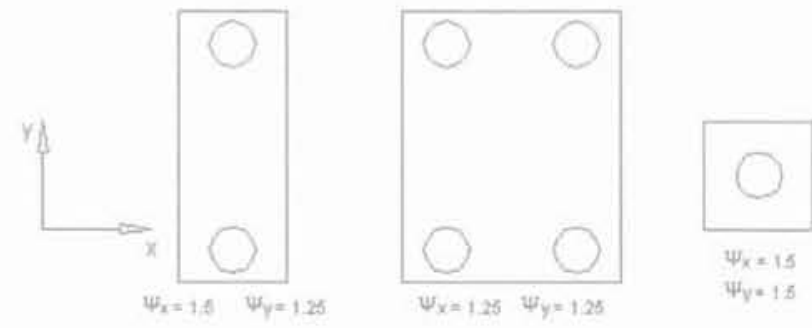

Les excentricités $\delta_{x}=\psi_{x} M_{x} / Q$ et $\delta_{y}=\psi_{y}, M_{y} / Q$ doivent vérifier:

$$
\delta_{x}<d_{x} \text { et } \delta_{y}<d_{y}
$$

\begin{tabular}{|l|c|c|}
\cline { 2 - 3 } \multicolumn{1}{c|}{} & $\mathrm{d}_{s}$ & $\mathrm{~d}_{\mathrm{y}}$ \\
\hline ELS quasi permanent & $<\mathrm{B} / 6$ & $<\mathrm{L} / 6$ \\
\hline ELS rare & $<\mathrm{B} / 4$ & $<\mathrm{L} / 4$ \\
\hline ELU & $<\mathrm{B} / 2$ & $<\mathrm{L} / 2$ \\
\hline
\end{tabular}

On se ramène alors à une répartition rectangulaire équivalente (Meyerhof) $q^{\prime}$ : 


\begin{tabular}{|c|c|c|}
\hline Étape & Principe général & $\begin{array}{l}\text { Cas particulier de données } \\
\text { pressiométriques }\end{array}$ \\
\hline 0 & $\begin{array}{l}\text { On vérifie d'abord la condition: } \\
\left.\text { in } S_{c o l} q_{n}+\left(S_{6}-n S_{c o l}\right) q_{\mu}^{\prime} / 3\right)>q_{5} \\
\text { avec } q_{s} \text { contrainte maximale admissible dans la colonne } \\
q_{u}^{\prime} \text { contrainte de rupture du sol sous charge centrée }\end{array}$ & (voir \$5.2) \\
\hline 1 & $\begin{array}{l}\text { On calcule le tassement } w_{,} \text {, sans traitement selon les règles } \\
\text { en vigueur; on détermine ainsi: } \\
k_{s}=q / w_{b}\end{array}$ & 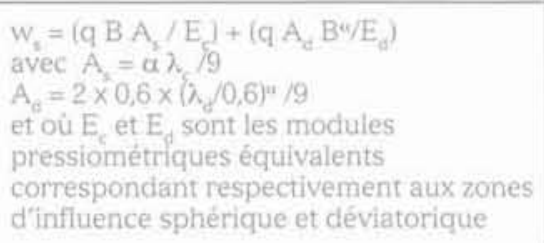 \\
\hline 2 & 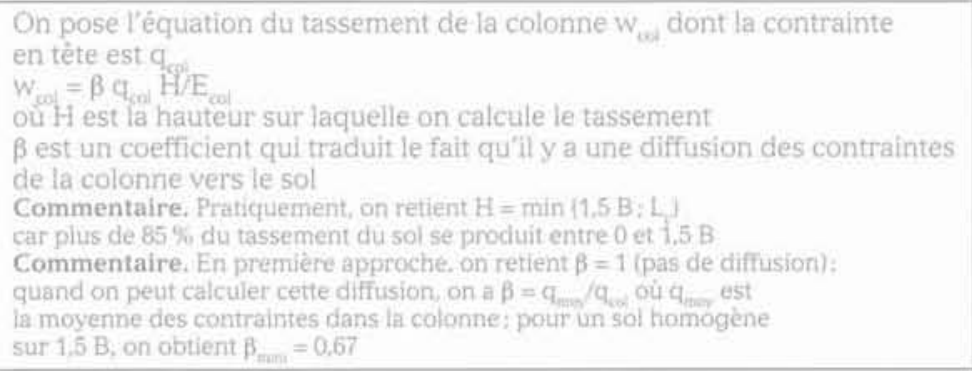 & \\
\hline 3 & $\begin{array}{l}\text { On en décluit l'expression de la raideur de la colonne: } \\
\mathrm{k}_{\mathrm{col}}=\mathrm{q}_{\mathrm{col}} / \mathrm{w}_{\mathrm{col}}=\mathrm{E}_{\mathrm{col}} /(\mathrm{\beta H})\end{array}$ & \\
\hline 4 & 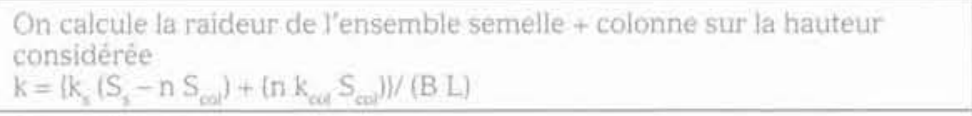 & \\
\hline 5 & $\begin{array}{ll}\text { On en déduit alors: } & \\
\text { - le tassement final appès traitement } & \mathrm{w}_{\mathrm{st}}=\mathrm{q} / \mathrm{k} \\
\text { - la contrainte sous la semelle } & \mathrm{q}_{\mathrm{s}}=\mathrm{w}_{\mathrm{s}} \mathrm{k}_{\mathrm{s}} \\
\text { - la contrainte dans la colonne } & \mathrm{q}_{\mathrm{col}}=\mathrm{w}_{\mathrm{d}} \mathrm{k}_{\mathrm{col}}\end{array}$ & \\
\hline 6 & 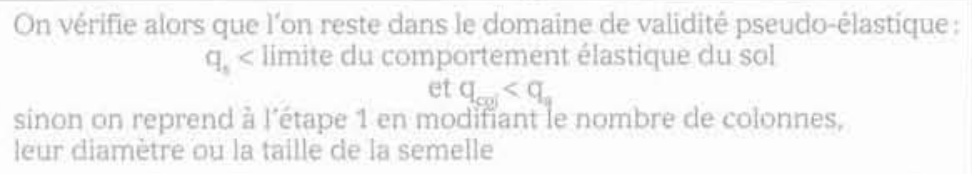 & $\begin{array}{l}\mathrm{q}_{4}<\mathrm{k} \mathrm{p}_{1} / 2+\mathrm{q}_{\mathrm{i}} \\
\mathrm{p}_{\mathrm{p}} / 2 \text { étant la valeur usuelle de limite } \\
\text { de validité des formules de tassement } \\
\text { selon Ménard }\end{array}$ \\
\hline
\end{tabular}

$\left.\mathrm{q}^{\prime}=\mathrm{q} \times \mathrm{B} \times \mathrm{L} /\left(\mathrm{B}-2 \delta_{\mathrm{y}}\right) \times\left(\mathrm{L}-2 \delta_{\mathrm{x}}\right)\right)$

On peut ensuite appliquer les méthodes simplifiées décrites au $\$ 5.5 .2 .1$ à la partie comprimée de la semelle.
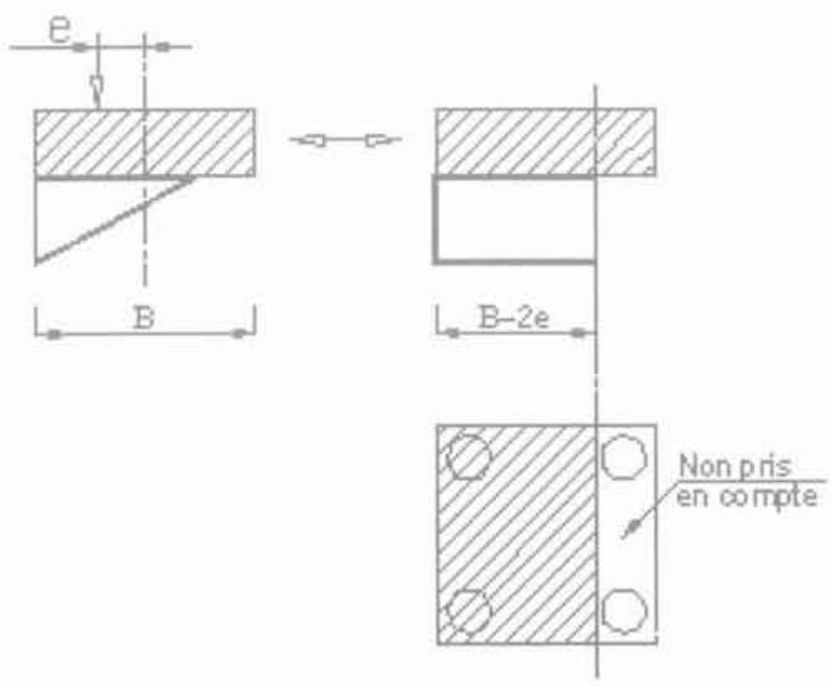

Commentaire. Pour qu'une colonne soit prise en compte dans le calcul, il faut vérifier qu'elle se situe dans la partie du sol entièrement comprimée. La majoration de 1,25 et/ou 1,5 permet de s'affranchir de cette vérification en prédimensionnement.

\section{$5 i 60$}

\section{Autres méthodes}

D'autres méthodes (sans majoration de $M_{x}$ et $M_{\text {f }}$ ) sont possibles; il faut alors vérifier:
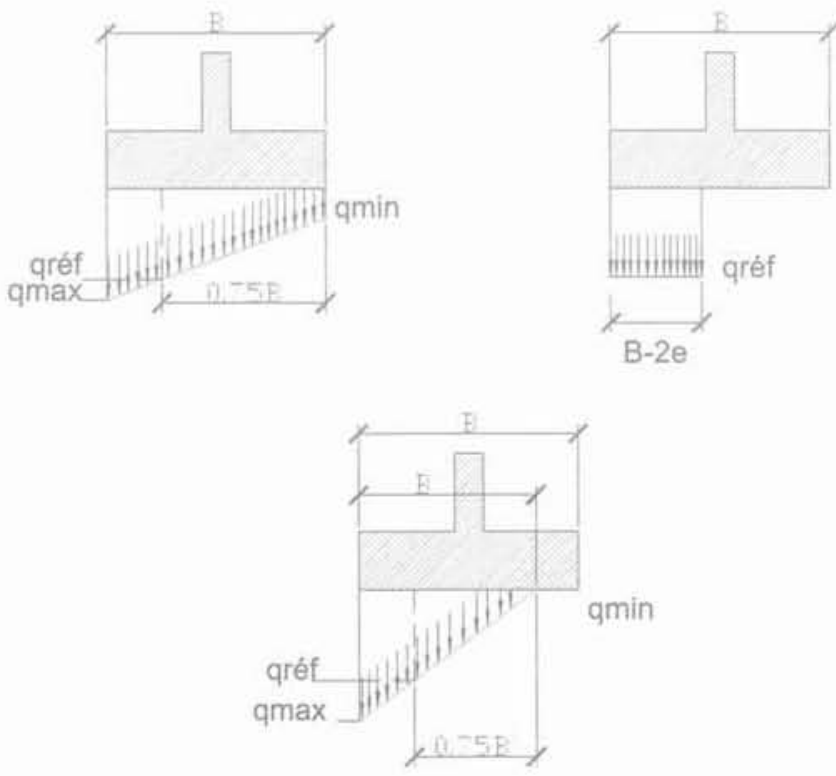
- que la contrainte de référence du sol après calcul [ $q^{\prime}$ $=(3 \mathrm{Pm}+\mathrm{pm}) / 4]$ appliquée sous la semelle reste dans le domaine de validité des formules de tassement.

Commentaire. Dans le cadre de la méthode pressiométrique. on doit vérifier:

1) $\mathrm{q}_{n+4}^{\prime}<\mathrm{k}^{*} \mathrm{p}_{1 / 2} / 2+\mathrm{q}^{\prime} \mathrm{o}$ (ct. $\$ 5.4 .2 .5$ )

2) que les contraintes dans les colonnes restent admissibles:

$\mathrm{q}_{\mathrm{col}}<\mathrm{q}_{\mathrm{a}}=2 \mathrm{p}$, à l'ELS

$q_{\text {coffui }}<q_{\text {afilu }}=4 \times p_{1} / 1,5$ à l'ELU

3) que le critère suivant de portance globale est vérifié avec: $\left.q^{\prime}=q^{*} B * L /\left(B-2 e_{2}\right) *\left(L-2 * e^{*}\right)\right]$,

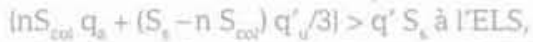

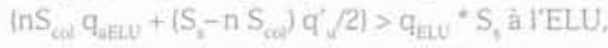

avec $\mathrm{q}_{\mathrm{a}}$ : contrainte maximale admissible dans la colonne á J'ELS, avec $q_{\text {in }}$ contrainte maximale admissible dans la colonne à I'ELU,

$q_{u}^{\prime}$ : contrainte de rupture du sol sous charge centrée,

4) que sous ELS rare $75 \%$ de la surface de la semelle reste comprimé, que sous ELS quasi permanent $100 \%$ de la surface reste comprimé, et que sous ELU $10 \%$ de la surface reste entièrement comprimé.

\section{7}

\section{Cas particulier des ouvrages en terre (chargement uniforme)}

Le calcul des colonnes ballastées sous les ouvrages en terre s'effectue comme pour le calcul des colonnes sous radiers et dallages soumis à une charge uniforme.

Ces ouvrages peuvent nécessiter la vérification de la stabilité vis-à-vis du glissement généralisé.

Dans ce cadre, il y a lieu de distinguer:

- le modèle initial correspondant au début de chargement sans aucun report de charge;

- le modèle progressif de transfert de charge (phase de drainage);

-le modèle final issu du report définitif de charge (fin de drainage).

Dans ce cadre, Prièbe (1978) propose des solutions d'homogénéisation qui, d'après Mitchel (1981), peuvent être appliquées à long terme (modèle final de transfert de charge) permettant d'obtenir un modèle homogène équivalent simplifié.

Cette approche simple ne permet pas de tenir compte du mécanisme de transfert de charge et peut sous-estimer le coefficient de sécurité lorsque le taux d'incorporation croit.

\section{8}

\section{Autres méthodes d'évaluation}

\section{Approche numérique aux éléments finis}

La méthode des éléments finis consiste à discrétiser le modèle géométrique $\alpha$ fini $»$ (sol + colonnes) en sousensembles de référence à partir de conditions aux limites en déplacement et en contraintes pour construire une matrice de rigidité globale.

On calcule ensuite, pour une loi de comportement donnée et pour chaque cas de chargement étudié, les déplacements, les déformations et les contraintes en tout point du modèle défini.

Cette méthode a l'avantage d'appréhender tous les paramètres du modèle retenu (déplacements, déformations, contraintes, iso-valeurs des paramètres et zones de plastification préalable).

Néanmoins, elle nécessite une attention particulière quant à la modélisation des colonnes et des conditions aux limites; les résultats dépendent fortement du modèle rhéologique et de ses paramètres géomécaniques (en particulier, module d'Young, coefficient de Poisson, Cc, Cs...), dont on rappelle qu'ils doivent être validés par le géotechnicien.

\section{Sas:}

\section{Méthodes d'homogénéisation}

Les méthodes d'homogénéisation présentent l'avantage de simplifier le milieu « composite sol + colonnes \# afin d'obtenir un modèle simple où le millieu traité est réduit à un milieu homogène équivalent.

La technique d'homogénéisation simplifiée a été utilisée d'abord par Prièbe (1978) pour les calculs de stabilité des ouvrages en terre sur sols mous traités par colonnes ballastées.

Commentaire. La méthode décrite au $\$ 5.5 .1$ fait partie de ces méthodes:

\section{6}

\section{Contrôles et réception}

\section{1}

\section{Contrôles en cours d'exécution}

\section{bith \\ Essais d'étalonnage}

Au démarrage de tout chantier de colonnes ballastées, l'entreprise doit effectuer des essais d'étalonnage destinés à valider le choix du matériel et à vérifier la conformité des réactions du sol avec les prévisions (profondeurs atteintes, consommations, soulèvements éventuels, influence des vibrations, etc.).

Dans le cas où l'étude de sol préalable ferait ressortir des hétérogénéités marquées dans les profondeurs, nature, caractéristiques des couches à traiter, il conviendra de procéder à des étalonnages dans chacune des différentes zones mises en évidence.

Ces essais seront réalisés de préférence au voisinage des sondages de l'étude géotechnique.

Commentaire. Les essais d'étalonnage sont des essais d'informations particuliers.

\section{arse}

\section{Essais d'information}

Pour une colonne sur 50 (avec un minimum de 3 par ouvrage), il sera fourni les paramètres d'exécution des colonnes ballastées: profondeur, énergie consommée, volume de matériau incorporé par mètre. 
Commentaire. Ces essais peuvent étre présentés sous la forme d'un enregistrement informatique, dans la mesure oú tous les paramètres sont renseignés.

\section{4 is}

\section{Attachements}

Ce document de synthèse donnera pour chaque colonne ses caractéristiques : date d'exécution, profondeur, énergie et volume consommé.

Il précisera en outre le matériel et le procédé utilisé.

Commentaire. Ces attachements peuvent être présentés sous la forme d'un enregistrement informatique, pour toutes les colonnes, de la profondeur, de l'énergie et du volume incorporé.

\section{2}

\section{Essais de réception}

Ces essais, qui sont à la charge, sauf stipulation contraire du marché, de l'entreprise qui exécute les travaux, comprennent selon le tableau ci-joint:

- contrôle du diamètre de la colonne;

- contrôle de la continuité, en cas de défaut d'enregistrement;

- contrôle de la compacité;

- essai de chargement.

Commentaire. Ces essais doivent être réalisés par un spécialiste agréé par le maître d'ouvrage.

Les résultats sont regroupés dans le dossier de récolement.

En outre, la tolérance d'exécution (cf. alinéa 4.9) est vérifiée à raison d'une colonne pour 80 pour les dallages et radiers par sondage et pour toutes les colonnes sous semelle,

Commentaire. Les colonnes réalisées hor's tolérances sont reportées sur un plan de récolement.

\section{9:4}

\section{Fréquence des essais}

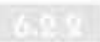

\section{Contrôle du diamètre}

Le dégarnissage sera réalisé sur des colonnes hors ouvrage, sur les différentes couches dont la profondeur permet la vérification.
Pour des raisons de faisabilité, ce dégagement se fera souvent en tête de colonne, sur une profondeur minimale de $1 \mathrm{~m}$ sous la plate-forme.

\section{$60 \mathrm{a}$ \\ Contrôle de continuité}

Le pénétromètre statique (norme NFP 94.113) est adaptè à cette vérification; le pénétromètre dynamique (norme NFP 94.114, type A) peut éventuellement être utilisé.

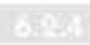

\section{Contrôle de compacité}

Il est réalisé à l'aide d'un pénétromètre statique (norme NFP 94.113). Ce contrôle doit descendre $1 \mathrm{~m}$ sous la base de la colonne, sauf refus sur la couche d'assise.

Pour être en accord avec les caractéristiques reprises dans l'alinéa 5,3 , la caractéristique minimale en tout point de l'axe de la colonne à partir de $1 \mathrm{~m}$ de profondeur doit être égale à: $\mathrm{q}_{\mathrm{cm}}=10 \mathrm{MPa}$.

Rappel de la définition de la résistance de pointe lissée $\left(q_{c m}\right)$ :

$$
q_{c m}=\frac{1}{4 a} \int_{D-a}^{D+3 a} q_{c}(z) d z
$$

a est égal $0,5 \mathrm{~m}$;

D est la profondeur à laquelle on considère la caractéristique;

$\mathrm{q}_{\mathrm{c}}(\mathrm{z})$ est la mesure obtenue, écrêtée à $1,3 \mathrm{q}_{\mathrm{cm}}$.

Commentaire. Ces essais peuvent présenter des difficultés de réalisation:

- blocage sur gros éléments du matériau constitutif de la colonne: - déviation du train de tige susceptible de sortir de la colonne.

Dans ce cas, l'entrepreneur propose un nouveau programme de contrôle.

Remarque; Les autres essais in situ habituels en géotechnique, à savoir l'essai au pressiomètre (norme NFP 94.110) avec essai tous les mètres et l'essai au pénétromètre dynamique de type A (au sens de la norme NFP 94.114), sont d'utilisation plus délicate dans les matériaux de type ballast.

Commentaire. Pour ètre représentatif. l'essai pressiométrique doit ètre réalisé à l'aide d'une sonde lanternée battue.

\begin{tabular}{|c|c|c|c|c|}
\hline \multirow[t]{2}{*}{$\begin{array}{l}\text { Fluide de } \\
\text { lançage }\end{array}$} & \multicolumn{2}{|c|}{ EAU } & \multicolumn{2}{|r|}{ AIR } \\
\hline & $\begin{array}{l}\text { Avec } \\
\text { enregistrement }\end{array}$ & $\begin{array}{l}\text { Sans } \\
\text { enregistrement }\end{array}$ & $\begin{array}{l}\text { Avec } \\
\text { enregistrement }\end{array}$ & $\begin{array}{l}\text { Sans } \\
\text { enregistrement }\end{array}$ \\
\hline $\begin{array}{l}\text { Contrôle } \\
\text { Diamètre }\end{array}$ & \multicolumn{4}{|c|}{1 par tranche de 50 colonnes jusqu'à 100 , minimum 3 au-delà } \\
\hline $\begin{array}{l}\text { Contrôle } \\
\text { Continuité }\end{array}$ & $\begin{array}{l}\text { Avec } \\
1 / 50\end{array}$ & $1 / 20$ & $\begin{array}{l}\text { Seulement en cas } \\
\text { d'anomalie }\end{array}$ & $1 / 50$ \\
\hline $\begin{array}{l}\text { Contrôle } \\
\text { Compacité }\end{array}$ & \multicolumn{4}{|c|}{$\begin{array}{c}1 / 80 \text { sous dallage ou radier }+1 / 20 \text { sous massif } \\
\text { avec un minimum de } 5\end{array}$} \\
\hline $\begin{array}{c}\text { Essai } \\
\text { Chargement* }\end{array}$ & $\begin{array}{l}1 \text { essa } \\
\text { et un autre }\end{array}$ & $\begin{array}{l}800 \mathrm{~m} \\
\text { che au-delà }\end{array}$ & $\begin{array}{l}1 \text { essai jusque } \\
\text { et un autr }\end{array}$ & $\begin{array}{l}\text { et } 400 \text { colonnes } \\
\text { ns au-delà }\end{array}$ \\
\hline
\end{tabular}

- Pour les chantiers de moins de $1000 \mathrm{~m}$ de colonnes ballastées par voie sèche ( $800 \mathrm{~m}$ par voie humide), on peut ne pas procéder à un essai de chargement mais, dans ce cas, la contrainte admissible sera minorée d'un coefficient 1.5 
Pour être en accord avec les caractéristiques reprises dans l'alinéa 5.3 , la caractéristique minimale en tout point de l'axe de la colonne à partir de $1 \mathrm{~m}$ de profondeur, en tenant compte des corrélations les plus récentes, doit être respectivement égale à :

$\mathrm{p}_{1}=1,2 \mathrm{MPa}$;

$\mathrm{q}_{\mathrm{d}}=10 \mathrm{MPa}$.

Dans le cas de valeurs inférieures, des justifications spécifiques devront être apportées.

\subsection{5}

\section{Essai de chargement}

C'est un essai de chargement à 1,5 fois la charge ELS de la colonne $Q_{N}$ sur une colonne de l'ouvrage.

L'essai de chargement nécessite la mise en place d'une semelle en tête de la colonne préalablement arasée sous le matelas de répartition. La surface de la semelle doit rester inférieure à 2,5 fois la section théorique de la colonne.

L'essai est de type à effort contrôlé, exécuté en compression; il consiste à mesurer l'enfoncement de la tête de la colonne (à l'aide de deux ou trois comparateurs) soumise à une charge verticale. paliers

Cette charge est appliquée progressivement en six

$\mathrm{Q}_{\mathbb{N}} / 4, \mathrm{Q}_{\mathrm{N}} / 2,3 \mathrm{Q}_{\mathbb{N}} / 4, \mathrm{Q}_{\mathrm{N}} / 5, \mathrm{Q}_{\mathrm{N}} / 4,3 \mathrm{Q}_{\mathrm{N}} / 2$

A chaque palier, les mesures de déplacement sont prises aux temps suivants :

$1,2,3,4,5,10,15,30,45$ et 60 minutes ou jusqu'à stabilisation des comparateurs:

Commentaire. La déformation est dite «stabilisèe » lorsque sa variation n'excède pas 2 centièmes de mm par minute.

Commentaire. Les premiers paliers peuvent donc avoir une durée de 30 minutes.

Le déchargement se fait en quatre paliers maintenus 5 minutes.

Le compte rendu d'essai comprend:

- le programme de chargement/déchargement avec les mesures des comparateurs en fonction du temps et de la charge;

- un graphique tassement/temps par paliers;

- un graphique tassement à la fin de chaque palier en fonction de la charge:

- un graphique de la pente de fluage en fonction de la charge.
L'essai est considéré comme probant si les deux critères suivants sont satisfaits:

- la charge critique ( (c) fluage») n'a pas été atteinte, - le tassement à la fin du palier de la charge de service $\left(Q_{N}\right)$ reste inférieur à celui estimé dans la note de calcul et compatible avec les tolérances imposées par la structure ou partie d'ouvrage.

\section{LEXIQUE}

Maille de référence. C'est la surface de l'emprise de louvrage divisée par le nombre de colonnes situées sous celui-ci.

Taux de substitution (ou pourcentage d'incorporation). C'est le rapport de la section de la colonne à la maille; il peut varier avec la profondeur.

Colonne ballastée pilonnée. Elle est obtenue par battage d'un tube obturé à sa base jusqu'à la profondeur recherchée. Le matériau est introduit par petites quantités en tête de tube et compacté au fur et à mesure par pilonnage en pied. Le tube est remonté petit à petit de sorte que le volume incorporé, toujours supérieur au gabarit du tube, est conforme au diamètre de calcul. Le diamètre du tube est choisi selon les caractéristiques du sol et de la colonne. Le poids du pilon est adapté à ce tube. L'énergie est modulée en faisant varier la hauteur de chute.

Colonne ballastée par voie sèche. Elle est obtenue par introduction dans le sol d'un vibreur radial jusqu'à la profondeur recherchée. Le matériau est refoulé dans le sol et compacté par ce vibreur, par passes successives. L'énergie mise en ceuvre et la vitesse de remontée sont adaptées au diamètre et à la compacité retenus dans le calcul. L'entraînement du vibreur peut être soit électrique, soit hydraulique. Par ailleurs, le remplissage s'effectue soit par la base, au moyen d'un tube latéral fixé au vibreur, soit par le haut.

Colonne ballastée par voie humide. Dito colonne ballastée sèche mais l'enfoncement est facilité par un lançage à I'eau (douce ou de mer).

Colonne ballastée à sas. Colonne ballastée dont le remplissage en matériau s'effectue par la base, avec application constante d'une pression d'air sur le ballast, sauf pendant le remplissage du tube d'apport.

Excentrement. Distance entre l'implantation théorique de la colonne et le centre de la colonne telle qu'exécutée. 


\section{ANNEXE I}

Colonnes ballastées en zone sismique

\section{Préambule}

Les indications décrites ci-après viennent en complément de celles décrites par ailleurs.

En cas de contradiction, la présente annexe prévaut.

\section{1}

\section{Dispositions constructives}

Des dispositions particulières doivent alors être retenues vis-à-vis du maillage et du matelas quand le géotechnicien a identifié:

- des couches liquéfiables dont il faut diminuer le potentiel de Liquéfaction par resserrement et densification du sol en place:

- des sols fins présentant un risque de baisse des caractéristiques mécaniques.

On tire les conclusions suivantes des retours d'expérience d'ouvrages spécifiques (bacs de stockage, réservoirs pour station d'épuration) fondés sur un maillage régulier de colonnes ballastées ayant subi des séismes de magnitude 6 à 7 et ne présentant pas de désordres dommageables:

- l'augmentation du taux de substitution minimal est adapté pour obtenir l'amélioration, d'une part, de la densité relative requise pour la prévention du risque de liquéfaction et, d'autre part, de la résistance au cisaillement:

- le débord de traitement est d'une rangée au minimum avec un nombre de rangées tel qu'il y a des colonnes sur une largeur de débord égale à la moitié de la profondeur de la base de la couche sensible au séisme.

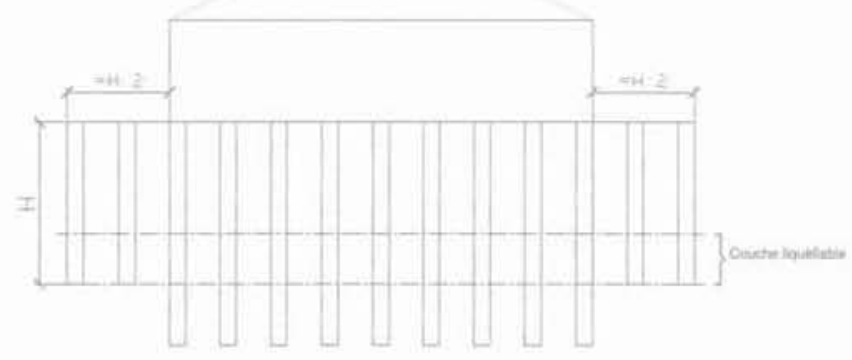

L'épaisseur du matelas de répartition granulaire est au minimum égale à $60 \mathrm{~cm}$ entre sous-face des radiers ou dallages et têtes de colonnes, pour faciliter la dissipation des pressions interstitielles.

Ces principes généraux doivent être adaptés au cas par cas moyennant justification pour tenir compte : - de la magnitude et/ou du zonage sismique;

- des caractéristiques géotechniques particulières;

- de la profondeur:

- du niveau du potentiel de liquéfaction;

- du type d'ouvrage et du mode de fondation.

\section{Dimensionnement}

\section{1}

\section{Critères de dimensionnement}

II faut calculer, en plus des cas « normaux $x$ :

- la baisse du potentiel de liquéfaction pour le ramener à une valeur admissible;

- le comportement des colonnes avec les caractéristiques de sol sous séisme et avec les cas de chargement qu'il génère.

\section{2}

\section{Hypothèses}

Le maître d'ouvrage et/ou ses représentants définissent les contraintes d'exploitation de l'ouvrage sous séisme: les sollicitations et les déformations admissibles:

Le géotechnicien doit donner les hypothèses concernant le sol, avec et sans séisme: stratigraphie, nature des sols, hydrologie, caractéristiques rhẻologiques et mécaniques de toutes les couches de sol.

Commentaire. Il faut en particulier disposer des hypothèses permettant de calculer les déformations sous actions cycliques et sous fortes sollicitations.

II peut fixer des objectifs d'amélioration.

\section{3}

\section{Contraintes dans les colonnes}

L'action du séisme intervient dans les combinalsons ELU sismiques.

La contrainte maximale de calcul qaELU dans la colonne est obtenue par application d'un coefficient de sécurité de 1,5 sur la contrainte verticale de rupture qr.

$$
\text { qaELU }=\text { qr } / 1,5
$$

Commentaire. Ceci revient à affecter un coefficient de 1,33 à la contrainte admissible ELS évaluée hors conditions de séisme:

$$
\text { qaELU }=1.33 \text { qa }
$$

\section{3.}

\section{Contrôles}

Il faut vérifier par des essais appropriés que l'amélioration du sol entre les colonnes a été obtenue après réalisation des colonnes.

Commentaire. Il est préférable d'utiliser le mème type d'essai avant et aprés réalisation dés colonnes ballastées. 


\section{ANNEXE II}

Dispositions types de colonnes ballastées sous semelles de fondation

NB. Les schémas sont établis pour des colonnes de diamètre $80 \mathrm{~cm}$. Toutes les cotes sont minimales et sont en $\mathrm{cm}$.

D'une manière générale, $d_{\min } \geq \operatorname{Max}(120 ; 1,5 \varnothing \mathrm{CB})$.

\section{DE 1 A 5 COLONNES}
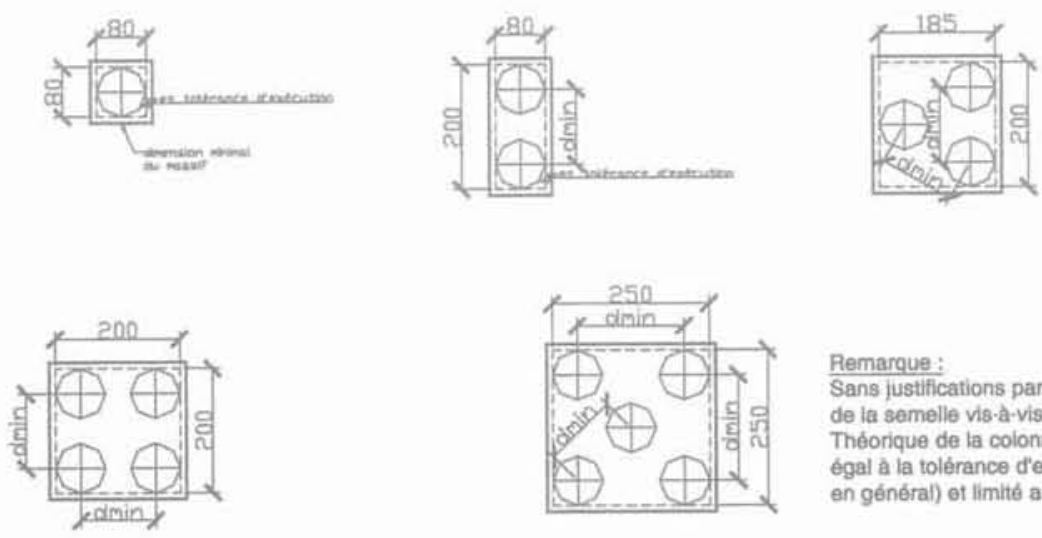

Remarque :

Sans justifications particulières, le débord de la semelle vis-à-vis du nu extérieur

Théorique de la colonne dolt être au moins

égal à la tolérance deexécution $(\mathrm{e}=20 \mathrm{~cm}$ en général) of limité au maximum a $40 \mathrm{~cm}$.

6 COLONNES ET PLUS
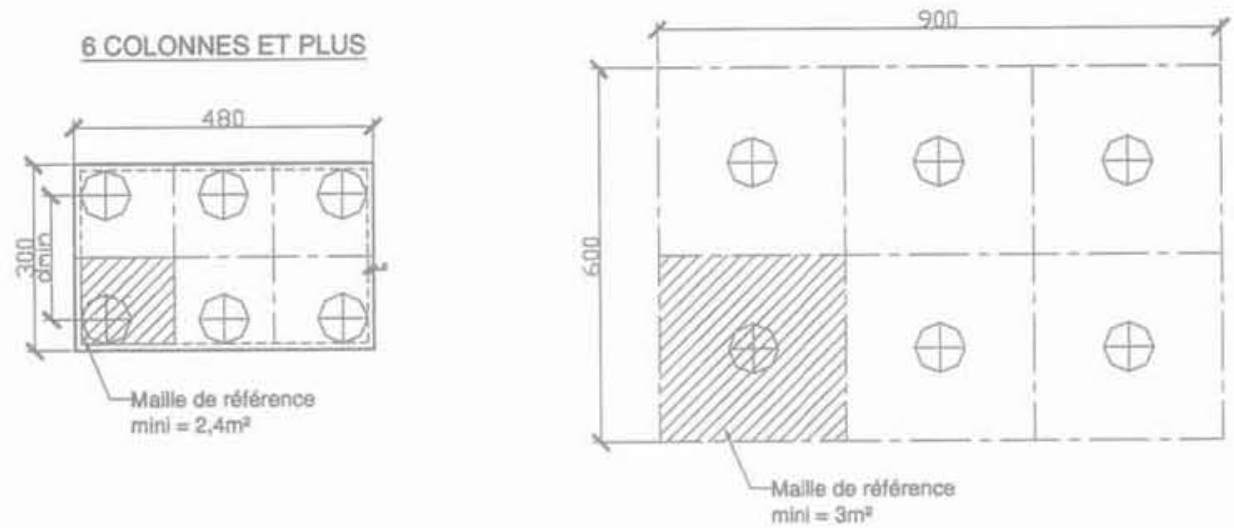

SEMELLE FILANTE
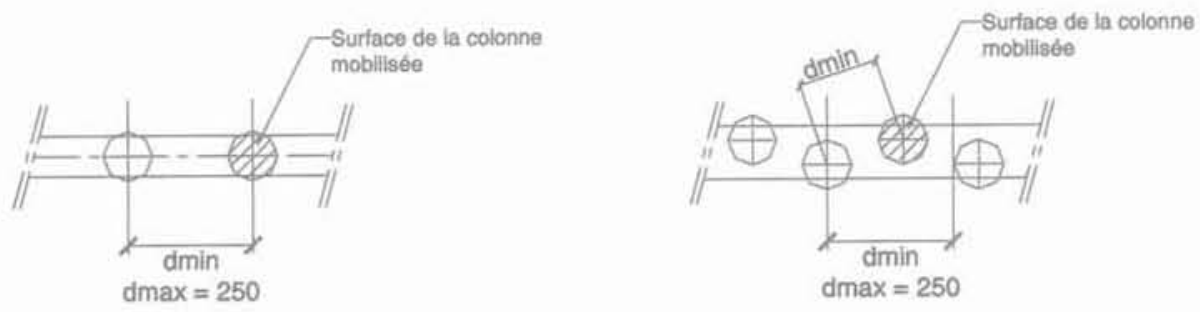

II est recommande orimplanter los colornes on

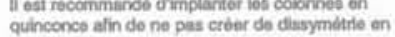
cas doxcentrament 